\title{
Adaptation and Validation of a Portuguese Version of the Sports Motivation Scale-II (SMS-II-P) Showing Invariance for Gender and Sport Type
}

Perceptual and Motor Skills

$0(0) 1-19$

(C) The Author(s) 2021

Article reuse guidelines: sagepub.com/journals-permissions DOI: I0.1 I77/003|5I252II039362 journals.sagepub.com/home/pms

@SAGE

\author{
F. Rodrigues ${ }^{1,2}\left(\mathbb{D}\right.$, L. Pelletier ${ }^{3}$, \\ M. Rocchi ${ }^{3}$, L. Cid Cid $^{2,4}$, D. Teixeira ${ }^{5}$ (D), \\ and D. Monteiro ${ }^{4,6}$ (D)
}

\begin{abstract}
In the present cross-sectional study, we adapted and examined the validity of a Portuguese version of the Sport Motivation Scale II (SMS-II-P) within a sample of I | 48 Portuguese athletes (women $=546$, men $=602$ ) with a mean age of I8.45 years $(S D=5.36)$, participating in a variety of sports (i.e., football, basketball, swimming, and athletics). We conducted confirmatory factor analysis, convergent and discriminant validity analysis, and multigroup analysis across participants' sport type (team and individual) and gender. We also examined the correlations between the SMS-II-P behavioral regulations and basic psychological needs satisfaction. The results supported that the SMS-II-P had good psychometric properties and was invariant across gender and sport type. The scale demonstrated good convergent and discriminant

\footnotetext{
'Life Quality Research Center, Santarém, Portugal

${ }^{2}$ Sport Science School of Rio Maior, Polytechnique Institute of Santarém, Rio Maior, Portugal

${ }^{3}$ University of Ottawa, Ottawa, Canada

${ }^{4}$ Research Center in Sport, Health and Human Development, Vila Real, Portugal

${ }^{5}$ University of Lusófona, Lisboa, Portugal

${ }^{6}$ ESECS, Polytechnique of Leiria, Leiria, Portugal

Corresponding Author:

F. Rodrigues, Av. Dr. Mário Soares n I I 0, 2040-4I3 Rio Maior, Portugal.

Email: ptfiliperodrigues@gmail.com
} 
validity, and the subscales achieved adequate internal consistency. Correlations between the six types of regulation measured in the SMS-II supported the distinction between autonomous and controlled behavioral regulations, and the correlations between these subscales and other measures of autonomy, competence, and relatedness satisfaction provided evidence of the self-determination continuum. Implications of this research for assessing Portuguese athletes and conducting future research are discussed.

\section{Keywords}

sport, motivation, self-determination theory, measurement, SMS-II

\section{Introduction}

Over the last 40 years, research guided by Self-Determination Theory (SDT; Ryan \& Deci, 2017) has shown that the ways athletes are motivated plays a critical role in the choices they make about (a) which activities to engage in, (b) which activities to persist in, (c) what degree of effort to expend, (d) which levels of performance to achieve, and (e) how their sport participation might contribute to their health and well-being (Hagger \& Chatzisarantis, 2007; Ntoumanis, 2012; Standage et al., 2019). According to SDT (Ryan \& Deci, 2017), athletes can be motivated by external factors such as reward systems, pressure from people in the sport context, evaluations, or the opinions they believe others may have of them. They can also be motivated by self-improvement, curiosity, and a desire for growth. More importantly, through its focus on fundamental distinctions between motives for behavior, SDT provides a comprehensive framework for understanding the interplay between the extrinsic forces, intrinsic motives, and needs that drive an individual's sport motivation.

\section{Self-Determination Theory}

SDT (Deci \& Ryan, 1985, 2000; Ryan \& Deci, 2017) proposed different types of motivation, or regulation types, that result from the satisfaction or frustration of basic psychological needs. Ryan and Deci (2017) highlighted the importance of these needs to foster motivation that is more autonomous or self-determined, compared to either motivation that is controlled by internal or external forces, or to no motivation (also called amotivation). The degree to which goals and behaviors are initiated and regulated through autonomous choice or as an expression of the self has a substantial and measurable impact on behavior, cognition, and experiences (Deci \& Ryan, 2000). According to SDT (Ryan \& Deci, 2017), the more internalized or self-determined the regulation of a behavior is, the more consistent one will be in acting in accordance with it, 
and the behavior will be autonomously self-regulated through time and across situations.

SDT outlined six types of behavior regulation that can be placed along a motivational continuum based on their degree of self-determination. According to Ryan and Connell (1989), these types of regulation form a simplex pattern such that the regulation types that are closely situated along the continuum tend to be more strongly related than the ones further away. At one end of the continuum, amotivation is positioned as the least self-determined form of behavior regulation as it represents a lack of any impetus to act (Deci \& Ryan, 2000). Hence, amotivation demarcates a state in which individuals perceive no relationship between their behavior and a desired outcome. external regulation, the least self-determined form of extrinsic motivation, is purely instrumental and serves as a means to obtain external rewards or to avoid negative consequences (e.g., punishments). Next, introjected regulation is not completely external; but rather, it proceeds through internal pressure and constraint, with external incentives turned inward but not truly accepted as one's own. Then, identified regulation refers to seeking goals that are personally valued or important such that the individual recognizes the relevance or significance of a behavior toward a value or personal standard. While significant to the individual, identified motives are not yet fully harmonized with the individual's overarching value system. Integrated regulation is the most self-determined form of extrinsic motivation. It occurs when personally endorsed behaviors become coherent, integrated, and assimilated within the self, fully aligning with the individual's other needs and values. Finally, intrinsic motivation is comprised of intrinsic regulation and represents the optimal form of behavioral regulation because activities are truly self-determined and pursued freely and out of enjoyment, generating a sense of satisfaction and capability. The intrinsically motivated individual perceives an internal locus of causality and engages in a given behavior out of a personal desire.

\section{Measuring Sport Motivation Using SDT}

To conduct sport-related motivation research grounded in SDT, past researchers developed a valid multi-dimensional measurement tool named the Sport Motivation Scale (SMS; Brière et al., 1995; Pelletier et al., 1995). The scale was built on the leading views of SDT at the time; it did not include any measure of integrated regulation but did include three intrinsic regulation subscales. In 2013, 18 years after the SMS was first published, Pelletier and colleagues revised the structure of the scale and the face validity of its items in order to: (a) add a validated integrated subscale; (b) replace problematic items; and (c) reduce the three intrinsic regulation subscales to one three-item factor. In addition, they reduced the SMS from four to three items per subscale, to make test administration briefer. Their revised SMS (SMS-II) showed stronger factor and 
construct validity and stronger reliability than the original version of the scale. They also showed that the integrated subscale represented a distinct construct from both identified regulation and intrinsic motivation, and that it was associated with higher satisfaction with life and vitality (Pelletier et al., 2013).

Since the publication of the SMS-II in 2013, the scale has generated considerable interest among motivational researchers (Standage et al., 2019; Standage \& Ryan, 2020). It has also been translated into Chinese (Li et al., 2018), French (Pelletier et al., 2019), Spanish (Granero-Gallegos et al., 2018; Pineda-Espejel et al., 2016), Turkish (Öcal \& Sakall1, 2018), and Portuguese (Júnior et al., 2014). This scale has also been subjected to rigorous invariance testing (Júnior et al., 2014; Li et al., 2018; Pelletier et al., 2019; Pelletier et al., 2013). Although some of the SMS-II items may not be fully invariant across specific sub-groups (e.g., Júnior et al. 2014), it is important to note that the psychometric properties of some of the items may be sample- or culture-specific, as reported by Pelletier et al. (2013). Since the SMS-II is a self-report instrument, it can be expected that the measure sometimes displays lower reliability or weaker support for the simplex pattern of correlations (Howard et al., 2017). In other words, in accordance with previous work on the measurement invariance between genders (Júnior et al., 2014), we feel that more studies are warranted to explore the application of the SMS-II in men and women athletes, as a means of exploring its applicability across groups with different characteristics, specifically in the Portuguese population, in which this measure has not been validated yet.

Previous studies have used the SMS-II exclusively with team sport athletes (e.g., Manouchehri et al., 2015) or both team and individual sport athletes (e.g., Júnior et al., 2014; Li et al., 2016; Ocal \& Sakalli, 2018; Pelletier et al., 2019; Pineda-Espejel et al., 2016). To the best of our knowledge, however, these studies have considered the SMS-II to be a valid instrument for both individual and team sport athletes, without proper psychometric testing. That is, these studies have combined athletes form different sport types (i.e., individual or team) and did not consider how behavioral regulations could differ depending on their sport participation. Hence, as Pelletier et al. (2017) pointed out, further examination of how the different forms of motivation assessed by the scale can reliably predict different outcomes such as sport participation, sport retention, performance, and other well-being indicators is needed. Finally, Pelletier et al. (2013) emphasized that construct validation of the SMS-II is an ongoing and continual process and, therefore, further examination of this measure is required to better understand the psychological processes underlying motivational processes that occur in sport.

\section{Current Research}

While a Portuguese version of the SMS-II has been adapted and validated with a sample of athletes from Brazil (Júnior et al., 2014), there are significant semantic 
differences and word meanings between Brazilian Portuguese and native Portuguese. Hence, while the Brazilian version of the SMS-II represents a reliable and valid scale in that culture, there remains a need for validating an SMSII scale for Portuguese athletes. Additionally, to the best of our knowledge, no study has examined whether the SMS-II is invariant across team and individual sports. Finally, there is a need for a new Portuguese version of the SMS-II to show invariance across gender as has been shown in other versions of the scale (e.g., Li et al., 2016; Pelletier et al., 2017; Pelletier et al., 2013). As such, in this study, we sought to adapt the SMS-II for athletes from Portugal (SMS-II-P) and to examine the validity and reliability of the SMS-II for both team and individual sport athletes and across gender. We expect that the SMS-II-P will demonstrate validity and reliability, as well as strong correlations internally between the six behavioral regulation subscales supporting the presence of a simplex pattern. For the correlations between the subscales and autonomy, competence, and relatedness satisfaction, we expect positive relationships between the more autonomous subscales and negative relationships for the more controlled subscales. We also anticipate that the SMS-II will be invariant across team and individual sports, as well as across gender.

If supported psychometrically, we expect the SMS-II-P to be highly beneficial for Portuguese athletes, as sport psychology is rapidly growing and developing in this country (Monteiro et al., 2019). This measure would be very helpful for coaches and practitioners who wish to optimize performance and well-being. While sport psychology, specifically using the SDT model, may still be in its infancy in Portugal, recent studies (Monteiro et al., 2020; Rodrigues et al., 2020) have suggested that assessing and examining athletes' motivation is crucial to facilitating a coach's engagement and connectedness with an athlete's well-being and self-determined motivation.

\section{Method}

\section{Participants}

We contacted different sports club managers and sports associations directors using a convenience sampling strategy to recruit participants for an online study. First, out of convenience, potential clubs and associations were screened and contacted individually during the pre-season (in Portugal this is between June and August). Second, after approval from the team managers and directors, athletes who agreed to partake this study signed an informed consent. Participating athletes completed an online questionnaire before training alone, but they were allowed to have assistance from the researcher when needed. The inclusion criterion to participate in this study were: (a) to be actively training in a sport club in Portugal during the time of data collection; (b) to be at least 15 years old (specialization years, Cotê et al., 2012); and (c) to consent to 
Table I. Sample Characteristics.

\begin{tabular}{lccccccc}
\hline Sample & $n$ & $M_{\text {age }}$ & $S D_{\text {age }}$ & $M_{\text {exp }}$ & $S D_{\text {exp }}$ & $M_{\text {train }}$ & $S D_{\text {train }}$ \\
\hline I. Team sports & 528 & 18.35 & 5.48 & 8.38 & 4.02 & 5.13 & 2.28 \\
2. Individual sports & 620 & 19.01 & 5.81 & 7.93 & 4.17 & 5.24 & 2.69 \\
3. Men & 602 & 18.76 & 5.47 & 8.63 & 4.13 & 4.99 & 2.13 \\
4. Women & 546 & 17.97 & 3.91 & 7.12 & 3.42 & 5.63 & 2.30 \\
\hline
\end{tabular}

Note. $\mathrm{n}=$ sample size; $M=$ Mean; $S D=$ Standard Deviation; exp = experience; train = weekly training sessions.

participating voluntarily in this study. Data were collected between April 2018 and March 2020. For this cross-sectional study, a sample of 1148 Portuguese athletes $($ women $=546$, men $=602)$ with a mean age of 18.45 years $(S D=5.36)$ met the inclusion criteria and agreed to be respondents for the validation of the SMS-II-P. The athletes represented a variety of sports (i.e., basketball, football, swimming, and athletics). Their sport experience ranged from 1 to 27 years $(M=8.03 ; S D=3.89)$, and they were training for an average of 5.05 sessions per week $(S D=2.17)$. We merged athletes playing basketball and football into a team sports group and those practicing individual sports (swimming and athletics) into an individual sports group. Detailed information for each sub-sample is displayed in Table 1. The researchers contacted different sports clubs and sports associations to recruit participants for an online study. However, due to the anonymous nature of the data, we are unable to provide response rates by clubs or associations.

Data collection was conducted in accordance with the Declaration of Helsinki and its later amendments. We obtained the approval of the Institutional Research Ethics Committee prior to data collection. All participants were informed about the main objective and goals of the study, and individuals 18 years old or older provided written informed consent before completing the questionnaires (see procedure details below). Underaged athletes who were interested in participating in this study had to receive approval from their legal guardian before participating.

\section{Procedures}

Procedures for this study involved both the development of an adapted Portuguese SMS-II (i.e., the SMS-II-P) and the validation of this instrument within a large sample of Portuguese athletes as described above. The development of the SMS-II-P and descriptions of other measures administered to the participants are described below in more details. Participants completed the online questionnaires individually before a sport training session in a quiet room (e.g., conference room). The average time taken to complete the multisection survey was 14.28 minutes $(S D=2.19)$. 


\section{Measures}

Sport Motivation Scale (SMS-II-P). As indicated previously, there is an existant Brazilian Portuguese version of the SMS-II (Júnior et al., 2014), but substantial differences between Brazilian and native Portuguese justify a separate Portuguese version of the SMS-II (i.e., our SMS-II-P). For example, the word "sport" in Brazilian Portuguese is "esportes" while in Portugal, the word for sport is "desporto". The syntax and grammar of these different ways of speaking Portuguese are also substantially different (see the Appendix 1 for a comparison of the two versions of the scale). Thus, we translated the original SMS-II scale (Pelletier et al., 2013 directly to Portuguese.

To translate and adapt the items from the SMS-II from its original language (English) into Portuguese, we followed Brislin's (1970) recommended methodological procedures and committee approach. This was a five-step process: (a) three experts with higher education in English-Portuguese languages did a preliminary translation of the scale; (b) this initial Portuguese version was reviewed by a panel of four scientific experts (a sport psychologist, a sport science researcher, an English-Portuguese teacher, and a psychologist) who generated a second version of the questionnaire that incorporated their suggested modifications; (c) a second panel of four other specialists joined the first panel to modify the second Portuguese version of the scale as needed in order to offer their consensual agreement that all items were properly translated; (d) a pilot sample of 40 bilingual college athletes $(28=$ men, $12=$ women $)$ aged 18 to 21 years $(M=19.09$; $S D=4.27$ ) with experience in psychometric testing determined whether the scale items were clear and correctly worded, made changes as necessary and offered a third version of the scale; and (e) two Portuguese teachers reviewed the final version for correct syntax, spelling, and grammar. This final Portuguese version of the SMS-II-P and the Brazilian version are both presented in Appendix 1 for comparison of the two versions of the scale in Portuguese.

Participants in the current study completed the 18-item SMS-II-P adapted to Portuguese to measure all six behavior regulation types accord to SDT. The participants responded to each item using a 7-point scale that ranged from 1 (Does not correspond at all) to 7 (Corresponds completely).

Basic Psychological Needs in Exercise Scale. The 12-item Basic Psychological Need in Exercise Scale was validated for the sport context by Monteiro et al. (2017) in order to measure the extent to which basic psychological needs were satisfied in sport. This scale was comprised of four items per subscale, measuring autonomy, competence, and relatedness satisfaction. Responses were provided on a five-point scale that ranged from 1 (I do not agree at all) to 5 (I completely agree). Original validation study (Vlachopoulos \& Michailidou, 2006) and cross-cultural invariance studies (e.g., Vlachopoulos et al., 2013) reported that the scale had satisfactory internal consistency $(\mathrm{a}>.70)$ and, recently, Rodrigues 
et al. (2021) demonstrated acceptable psychometric proprieties in several samples (i.e., athletes, exercisers, and students).

\section{Data Analysis Plan}

Construct Validity and Reliability. Analyses were performed in Mplus 7.4 (Muthén \& Muthén, 2010). We used the Robust Maximum Likelihood estimator to correct for non-normality bias. As previous theoretical (Ryan \& Deci, 2017) and empirical (Pelletier et al., 2013) studies have supported the six-factor measurement model, we tested the correlated six-factor model using confirmatory factor analysis (CFA). We used the Full Information Robust Maximum Likelihood (FIML) method to handle a small amount of missing data at the item level (missing at random $=3 \%$ ), as proposed by Enders (2010). Due to the oversensitivity of the chi-square statistics on large samples and the model complexity (Hair et al., 2019), we considered several common goodness-of-fit indices to assess model fit, namely: Tucker-Lewis Index (TLI), Comparative Fit Index (CFI), Root Mean Square Error of Approximation (RMSEA) and its respective Confidence Interval at 90\% (CI 90\%), and Standardized Root Mean Residual (SRMR). For CFI and TLI, values $\geq .90$ are typically interpreted to reflect acceptable fit and for SRMR and RMSEA, values of $\leq .80$ are indicative of adequate fit to the data (Hair et al., 2019; Marsh et al., 2004). Analysis of the individual items should display significant loadings on the target factor, with weights greater than .50 and significant $(p<.05)$, and they should explain at least $25 \%$ of the variance (Hair et al., 2019). For the assessment of internal consistency, composite reliability coefficients were calculated for the subscale scores, and values $\geq .70$ were considered acceptable (Raykov et al., 2016).

Sample Size. Kline (2016) recommended a 5:1 minimum ratio of participants per parameter to be estimated. With 69 parameters to be estimated in the correlated six-factor model, our sample of 1148 participants far exceeded this rule of thumb (345 minimum). Additionally, Hair et al. (2019) recommend that factor analyses should be carried out on samples larger than 300, which is also below the current sample size in this study. Finally, G*Power v3.1 software (Faul et al., 2009) was used as a complementary sample size analysis to calculate the minimum required sample size for this study. The following inputs were used: anticipated effect size of $\mathrm{f} 2=0.01, \alpha=0.05$, and statistical power $=0.95$. The software suggested a minimum of approximately 300 participants, which provided additional support that current sample size is acceptable.

Measurement Invariance. First, we examined the correlated six-factor model for team sports, individual sports, and gender samples separately. Then, we measured several levels of measurement invariance according to recommendations from Cheung and Rensvold (2002). These levels are: configural invariance (i.e., 
factor structure is the same between groups; same items associated with the same factors); weak factorial invariance (i.e., factor structure and factor loadings are equal between groups); strong invariance (i.e., item factor structure, factor loadings, and item intercepts are equal between groups), and strict factorial invariance (i.e., item factor structure, factor loadings, item intercepts, and item residuals are equal between groups). Model comparisons were made according to several recommendations, specifically: (a) differences in CFI and TLI $\leq 0.010$ (Marsh et al., 2014), and (b) differences in SRMR and RMSEA $\leq 0.015$ (Cheung \& Rensvold, 2002).

Convergent and Discriminant Validity and Correlational Analysis. The Average Variance Extracted (AVE) and the comparison between the square root of the AVE and squared correlations were used to investigate convergent and discriminant validity, respectively. AVE is an established approach to test convergent validity (Hair et al., 2019). Constructs are identified as distinct when the square root of the AVE value is larger than the correlation between the two constructs and when the AVE for each construct is above 0.50 displaying discriminant validity (Hair et al., 2019). Correlational analyses with latent variables were conducted, where each motivational type and each need were considered separately. The level of significance for the correlations was set at $p<.05$.

\section{Results}

\section{Construct Validity and Reliability}

The six-factor CFA models displayed adequate fit to the data in all samples, as well as in the full sample of participants as seen in Table 2. While TLI was below the cutoff in the individual sports sample, it was close to achieving acceptable values. The SRMR and RMSEA were also below the acceptable cutoffs.

Table 2. Summary of Goodness-of-Fit Indexes for the Tested Models.

\begin{tabular}{|c|c|c|c|c|c|c|c|}
\hline Model & $\chi^{2}$ & $d f$ & $\mathrm{CFI}$ & TLI & SRMR & RMSEA & $90 \% \mathrm{Cl}$ \\
\hline $\begin{array}{l}\text { I. Six-correlated factor model - team } \\
\text { sports }\end{array}$ & $785.745^{*}$ & 120 & .922 & .900 & .057 & .077 & $.073, .082$ \\
\hline $\begin{array}{l}\text { 2. Six-correlated factor model - } \\
\text { individual sports }\end{array}$ & $821.035^{*}$ & 120 & .924 & .901 & .056 & .076 & $.069, .079$ \\
\hline 3. Six-correlated factor model - men & $1263.457^{*}$ & 120 & .914 & .905 & .053 & .074 & $.062, .080$ \\
\hline 4. Six-correlated factor model - women & $961.101 *$ & 120 & .910 & .901 & .058 & .079 & $.065, .084$ \\
\hline $\begin{array}{l}\text { 5. Six-correlated factor model - total } \\
\text { sample }\end{array}$ & $1368.304 *$ & 120 & .913 & .903 & .055 & .078 & $.07 \mathrm{I}, .086$ \\
\hline
\end{tabular}

$*_{p}<0.00$ I. 
Table 3. Factor Loadings, Uniqueness, and Composite Reliability Coefficients of the Six Correlated Models.

\begin{tabular}{|c|c|c|c|c|c|c|c|c|}
\hline & \multicolumn{2}{|c|}{$\begin{array}{c}\text { Team sport } \\
\text { sample }\end{array}$} & \multicolumn{2}{|c|}{$\begin{array}{l}\text { Individual sport } \\
\text { sample }\end{array}$} & \multicolumn{2}{|c|}{ Women sample } & \multicolumn{2}{|c|}{ Men sample } \\
\hline & $\lambda$ & SE & $\lambda$ & SE & $\lambda$ & SE & $\lambda$ & SE \\
\hline Amotivation & .86 & & .84 & & .85 & & .86 & \\
\hline Item 2 & $.79 *$ & .03 & $.78 *$ & .03 & $.78 *$ & .01 & $.77 *$ & .02 \\
\hline Item 10 & $.84 *$ & .02 & $.79 *$ & .03 & $.83 *$ & .01 & $.82 *$ & .02 \\
\hline Item I3 & $.82 *$ & .03 & $.83 *$ & .03 & $.82 *$ & .01 & $.85 *$ & .02 \\
\hline External regulation & .90 & & .89 & & .91 & & .89 & \\
\hline Item 5 & $.89 *$ & .02 & $.87 *$ & .03 & $.88 *$ & .01 & $.90 *$ & .01 \\
\hline Item 8 & $.78 *$ & .03 & $.77 *$ & .03 & $.77 *$ & .01 & $.74 *$ & .02 \\
\hline Item 15 & $.93 *$ & .01 & $.90 *$ & .01 & $.91 *$ & .01 & $.90 *$ & .01 \\
\hline Introjected regulation & .80 & & .82 & & .81 & & .82 & \\
\hline Item I & $.69 *$ & .03 & $.7 I *$ & .03 & $.72 *$ & .02 & $.74 *$ & .02 \\
\hline Item 7 & $.74 *$ & .03 & $.76 *$ & .03 & $.72 *$ & .01 & $.76 *$ & .02 \\
\hline Item 16 & $.82 *$ & .03 & $.85 *$ & .02 & $.80 *$ & .01 & $.82 *$ & .02 \\
\hline Identified regulation & .70 & & .74 & & .71 & & .72 & \\
\hline Item 6 & $.63 *$ & .05 & $.69 *$ & .04 & $.62 *$ & .02 & $.65 *$ & .03 \\
\hline Item 12 & $.69 *$ & .04 & $.65 *$ & .04 & $.64 *$ & .02 & $.67 *$ & .03 \\
\hline Item 18 & $.72 *$ & .04 & $.75 *$ & .03 & $.75 *$ & .02 & $.72 *$ & .03 \\
\hline Integrated regulation & .77 & & .76 & & .76 & & .77 & \\
\hline Item 4 & $.62 *$ & .05 & $.65 *$ & .03 & $.61 *$ & .02 & $.62 *$ & 03 \\
\hline Item II & $.85 *$ & .02 & $.80 *$ & .03 & $.82 *$ & .01 & $.84 *$ & .02 \\
\hline Item I4 & $.7 I^{*}$ & .03 & $.69 *$ & .04 & $.72 *$ & .02 & $.7 I *$ & .02 \\
\hline Intrinsic regulation & .83 & & .86 & & .84 & & .85 & \\
\hline Item 3 & $.86 *$ & .02 & $.89 *$ & .03 & $.89 *$ & .01 & $.88 *$ & .02 \\
\hline Item 9 & $.88 *$ & .02 & $.88 *$ & .02 & $.87 *$ & .01 & $.90 *$ & .01 \\
\hline Item 17 & $.63 *$ & .02 & $.66 *$ & .04 & $.61 *$ & .03 & $.64^{*}$ & .03 \\
\hline
\end{tabular}

Note. $\lambda=$ standardized factor loadings; $\mathrm{SE}=$ Standard Errors; composite reliability coefficients are in italic. $*_{p}<0.01$.

Analyses on the correlated six-factor models (see Table 3) revealed that all item loadings on the target factor were greater than .50 and loaded significantly at $p<.01$. Additionally, responses to each behavioral regulation were found to be internally consistent as all factors within the correlated six-factor CFA model had composite reliability coefficient scores equal or above .70. Composite scores ranged between .70 (identified regulation in the female sample) and .90 (external regulation in the male sample).

\section{Measurement Invariance}

The correlated six-factor model was employed for measurement invariance analyses across team and individual sports, and across gender, since the model fit the 
Table 4. Multigroup Analysis Using the Six-Correlated Factor Model Between Sports and Gender.

\begin{tabular}{lcccccccccc}
\hline \multicolumn{1}{c}{$\chi 2$} & df & CFI & $\Delta$ CFI & TLI & $\Delta$ TLI & SRMR & $\Delta$ SRMR & RMSEA & $\Delta R M S E A$ \\
\hline Sports & & & & & & & & & & \\
Configural & $987.789 *$ & 262 & .912 & - & .903 & - & .064 & - & .069 & - \\
Weak & $1236.963^{*}$ & 274 & .910 & .002 & .899 & .004 & .060 & .004 & .072 & .003 \\
Strong & $1301.203^{*}$ & 288 & .906 & .006 & .898 & .005 & .069 & .005 & .075 & .006 \\
Strict & $1979.364^{*}$ & 302 & .901 & .011 & .888 & .005 & .072 & .006 & .076 & .007 \\
Gender & & & & & & & & & & \\
Configural & $969.368^{*}$ & 262 & .911 & - & .903 & - & .063 & - & .072 & - \\
Weak & $1103.86 I^{*}$ & 274 & .906 & .005 & .901 & .002 & .069 & .004 & .080 & .008 \\
Strong & $1245.369 *$ & 288 & .906 & .005 & .898 & .005 & .073 & .010 & .081 & .009 \\
Strict & $1999.736^{*}$ & 302 & .899 & .012 & .895 & .008 & .075 & .012 & .085 & .013 \\
\hline
\end{tabular}

Note. $\Delta=$ differences.

$* p<0.001$.

data in each sample individually. Next, we tested configural, weak, strong, and strict levels of invariance. Statistical differences among constrained and nested models were below accepted cutoffs, except for strict invariance criteria in the multigroup analysis between gender. Nonetheless, since this criterion seems to be optional and rarely achieved in social sciences (Byrne, 2016), it is possible to confirm that the SMS-II 18-item factor structure showed good levels of invariance across team and individual sports, and gender (for more details see Table 4).

\section{Convergent and Discriminant Validity and Correlational Analysis}

Convergent validity was achieved as the AVE scores were above 0.5 as seen in Table 5. Discriminant validity was evaluated by comparing the squared correlations in each latent variable against the AVE scores in each latent variable. According to the squared correlations and AVE scores in Table 5, all factors demonstrated adequate discriminant validity. The correlations of the correlated six-factor model showed significant associations, as theoretically expected, in all samples under analysis, specifically: (a) self-determined regulations were positively correlated with each other and negatively correlated with nonself-determined regulations, and (b) non-self-determined regulations were positively correlated with each other.

For the correlational analysis with the measures of the three basic needs, the SMS-II-P factors were specified as latent variables. Overall, these findings provided support for our hypothesis: autonomy, competence, and relatedness satisfaction were positively associated with the autonomous types of regulations, 
Table 5. Average Variance Extracted and Correlations.

\begin{tabular}{|c|c|c|c|c|c|c|c|c|}
\hline & AVE & $\sqrt{A V E}$ & I & 2 & 3 & 4 & 5 & 6 \\
\hline I. Amotivation & .69 & .83 & I & .58 & .50 & .07 & .20 & .18 \\
\hline 2. External regulation & .77 & .88 & $.76^{* *}$ & I & .48 & .10 & .34 & .37 \\
\hline 3. Introjected regulation & .60 & .77 & $.7 I^{* *}$ & $.69 * *$ & I & .05 & .20 & .44 \\
\hline 4. Identified regulation & .54 & .73 & $-.26 * *$ & $-\left..3\right|^{* *}$ & -.23 ** & I & .41 & .27 \\
\hline 5. Integrated regulation & .56 & .75 & $-.45^{* *}$ & $-.58^{* *}$ & $-.45^{* *}$ & $.64^{* *}$ & I & .49 \\
\hline 6. Intrinsic regulation & .66 & .81 & $-.42 * *$ & $-.6 \mathrm{I}^{* *}$ & -.66 ** & $.52 * *$ & $.70 * *$ & I \\
\hline
\end{tabular}

Note. $A V E=$ Average Variance Extracted; the zero-order correlations appear below the diagonal; the squared correlations appear above the diagonal.

**p $<0.05$.

Table 6. Correlations Between Needs and Motivational Regulations.

\begin{tabular}{llll}
\hline & Autonomy & Competence & Relatedness \\
\hline Amotivation & $-.11^{* *}$ & $-.13^{* *}$ & $-.19^{* *}$ \\
External regulation & $-.12^{* *}$ & $-.14^{* *}$ & $-.18^{* *}$ \\
Introjected regulation & $-.07^{*}$ & $-.10^{* *}$ & $-.20^{* *}$ \\
Identified regulation & $.26^{* *}$ & $.21^{* *}$ & $.22^{* *}$ \\
Integrated regulation & $.34^{* *}$ & $.25^{* *}$ & $.25^{* *}$ \\
Intrinsic regulation & $.22^{* *}$ & $.20^{* *}$ & $.18^{* *}$ \\
\hline
\end{tabular}

$* p<0.05 . * * p 0.01$

and they were negatively correlated with the controlled regulations, as theoretically expected. For detailed information see Table 6.

\section{Discussion}

The present study objectives were two-fold: (a) translate and adapt the SMS-II to the Portuguese language, and (b) analyze its psychometrics properties (factorial validity with gender and sports invariance analyses, reliability and construct validity) of the SMS-II-P with a sample of Portuguese speaking participants from Portugal. Our main findings were as follows.

Like the original SMS-II, our new SMS-II-P included 18 items that measured the six regulation types proposed by SDT - intrinsic, integrated, identified, introjected and external regulation, and amotivation. Overall, the SMS-II-P structure, including its factor structure, its tests of invariance for gender and across team and individual sports, its internal consistency, and its fit with a simplex model, were generally supported. Also, we examined the construct validity of the scale through a series of correlations between the different subscales and psychological need satisfaction. These analyses provided good support for the validity and the reliability of the scale and suggested that the 
psychometric properties of the SMS-II-P are similar to the psychometric properties of the SMS-II. It should be noted that a complete assessment of the psychometric properties of the SMS-II-P, such as test-retest reliability, and how well the different forms of motivation assessed by the scale predict different outcomes like sport participation, sport retention, and sport performance over time will necessitate additional research.

Multigroup invariance testing across gender and type of sports revealed that the six-factor structure of the SMS-II-P adequately represented the motivational orientations as defined by SDT for both men and women athletes, as well as for athletes in both individual and team sports. That is, strong configural invariance was obtained across all groups. Moreover, overall fit indices revealed that multigroup models comprising factor loadings and intercept constraints displayed a satisfactory adjustment to sample covariance. Invariance of factor loadings and/ or intercepts across several groups also qualifies as a very complex covariance structure analysis. In this context, it is reasonable to expect significant differences regarding item residuals between groups (i.e., strict invariance) could be detected (Byrne, 2016; Cheung \& Rensvold, 2002) as it was between men and women.

Correlations between the six types of behavior regulation measured in the SMS-II-P provided evidence of the distinctions between autonomous and controlled behavioral regulations, and the correlations between these subscales and other measures of autonomy, competence, and relatedness satisfaction provided evidence of the self-determination continuum. As we expected from SDT theory (Ryan \& Deci, 2017), autonomous regulations were positively correlated with each other, whereas controlled regulations were negatively correlated with autonomous regulations. These results support previous evidence on the validation of the SMS-II (Pelletier et al., 2017; 2013), indicating that this measure provides adequate evidence of the distinctiveness across behavioral regulations, and that athletes are able to distinguish autonomous and controlled forms of motivation. Additionally, these results are consistent with those described by Howard et al. (2017) related to the simplex pattern specifically in sport motivation research, in which regulations that are closer to each other show stronger correlations than those that are farther apart.

Correlations between behavioral regulations and autonomy, competence, and relatedness satisfaction provided evidence of the self-determination continuum (Ryan \& Deci, 2017). That is, intrinsic motivation, integrated, and identified regulations were positively correlated with all three basic psychological needs whereas controlled regulations were negatively correlated with autonomy, competence, and relatedness satisfaction. This was also expected, both theoretically (Ryan \& Deci, 2017) and empirically (Monteiro et al., 2020), as the satisfaction of all three needs provides overall well-being and adaptive outcomes as behavior is more internalized and personally accepted by the individual. 
The results of this study should help researchers and practitioners investigate behavioral regulations, grounded in SDT, among Portuguese athletes. Furthermore, the SMS-II-P may be useful for examining how coaches' interpersonal behaviors relate to the different types of motivation (Rocchi \& Pelletier, 2018). In turn, both factors could be included in studies to examine how they can optimize sport performance (Ntoumanis, \& Mallett, 2014), long-term participation (Sarrazin et al., 2002), as well as increase goal achievement, and overall well-being (Ryan \& Deci, 2017).

The present study also expands the support for the multi-dimensional structure of sport motivation, as defined by SDT, by showing that the SMS-II has very good psychometric properties in a different culture. Additionally, the results provide further support for the cross-cultural generalizability of SDT to the Portuguese culture more broadly, as well as the sport domain within that culture more specifically.

As suggested by Pelletier et al. (2019), the assessment of different and distinct behavioral regulations in sport may prove fruitful for examining the role of the specific types of motivation in the quality of sport experience and their roles in the global functioning of athletes in other life domains. The inclusion of the integrated subscale should be helpful for examining the extent to which athletes who show higher levels of self-determined or autonomous functioning attempt to regulate other activities in their life that could be important for optimal functioning in sport, such as the adoption of an healthy diet, good sleeping behavior, and good sportsmanship. The scale could also be used to determine whether athletes with levels of integrated regulation show more resilience to adversity (e.g., injuries, defeat or a set-back in a performance) or more positive sport outcomes (e.g., more concentration, better performance, sustained engagement) and less negative psychological outcomes (e.g., anxiety and stress).

\section{Limitations, and Directions for Future Research}

The present study was limited by its cross-sectional design and relative age homogeneity among participants. Additionally, we used a convenience sample of motivated and experienced participants who may have shown more interest in our study than most young athletes. Even though the sample was relatively large, future studies should aim to recruit a wider age of participants and seek to represent a more varied distribution of sport abilities. Also, it would be interesting to expand our knowledge of the functional equivalency of the SMS-II-P by testing its invariance across time in future longitudinal research projects comprising several data waves.

Finally, now that several versions of the SMS-II have been validated in different languages and in different cultures, it would be interesting to examine whether the scale, and the motivational types that the scale measures, are invariant across cultures. These analyses could include participants from different 
countries that speak different languages, participants from different countries that speak the same language, or participants from different cultures broadly defined as, for example, participants from Western cultural groups compared to Eastern cultural groups.

\section{Conclusion}

In summary, the present findings provide support for the validity and reliability of the SMS-II for native Portuguese athletes (SMS-II-P). Although the present results need to be replicated with other Portuguese samples, these findings support that the SMS-II-P represents a psychometrically sound scale that can be used in future research with Portuguese participants.

\section{Declaration of Conflicting Interests}

The author(s) declared no potential conflicts of interest with respect to the research, authorship, and/or publication of this article.

\section{Funding}

The author(s) disclosed receipt of the following financial support for the research, authorship, and/or publication of this article: F. R. was supported by the national funds through the Portuguese Foundation for Science and Technology, I.P., under the project UIDP $/ 04748 / 2020$. L. C. and D. M. were supported by national funds through the Portuguese Foundation for Science and Technology, I.P., under the project UID04045/2020.

\section{ORCID iDs}

F. Rodrigues (D) https://orcid.org/0000-0003-1327-8872

L. Cid (D) https://orcid.org/0000-0001-8156-3291

D. Teixeira (D) https://orcid.org/0000-0003-4587-5903

D. Monteiro (D) https://orcid.org/0000-0002-7179-6814

\section{Supplementary Material}

Supplemental material for this article is available online.

\section{References}

Brière, N., Vallerand, R., Blais, M., \& Pelletier, L. (1995). Development and validation of a scale on intrinsic and extrinsic motivation and lack of motivation in sports: The scale on motivation in sports. International Journal of Sport Psychology, 26(4), 465-489.

Brislin, R. (1970). Back-translation for cross-cultural research. Journal of Cross-Cultural Psychology, 1(3), 185-216. https://doi.org/10.1177/135910457000100301

Byrne, B. (2016). Structural equation modeling with AMOS. Basic concepts, applications, and programming (3rd ed). Taylor \& Francis Group, LLC. 
Cheung, G., \& Rensvold, R. (2002). Evaluating goodness-of-fit indexes for testing measurement invariance. Structural Equation Modeling: A Multidisciplinary Journal, 9(2), 233-255. https://doi.org/10.1207/s15328007sem0902_5

Cotê, J., Murphy-Mills, J., \& Abernethy, B. (2012). The development of skill in sport. In H. Hodges \& A. M. Williams (eds.)., Skill acquisition in sport: research, thery and practice (pp. 269-286). New york: Routledge

Deci, E., \& Ryan, R. (2000). The "what" and "why" of goal pursuits: Human needs and the self-determination of behavior. Psychological Inquiry, 11(4), 227-268. https://doi. org/10.1207/S15327965PLI1104_01

Deci, E., \& Ryan, R. (1985). Intrinsic motivation and self-determination in human behavior. Plenum. https://doi.org/10.1007/978-1-4899-2271-7

Enders, C. (2010). Applied missing data analysis. The Guilford Press.

Faul, F., Erdfelder, E., Buchner, A., \& Lang, A. (2009). Statistical power analyses using $\mathrm{G}^{*}$ Power 3.1: Tests for correlation and regression analyses. Behavior Research Methods, 41(4), 1149-1160. https://doi.org/10.3758/BRM.41.4.1149

Granero-Gallegos, A., Gómez-López, M., González-Hernández, J., Baena-Extremera, A., \& Ortiz-Camacho, M. (2018). Spanish adaptation and psychometric properties of the Sport Motivation Scale-II with high school physical education students. International Journal of Environmental Research and Public Health, 15, 2768. https:// doi.org/10.3390/ijerph15122768

Hair, J., Black, W., Babin, B., \& Anderson, R. (2019). Multivariate data analysis (8th ed.). Pearson Educational.

Hagger, M., \& Chatzisarantis, N. (2007). Intrinsic motivation and self-determination in exercise and sport. Human Kinetics.

Howard, J. L., Gagné, M., \& Bureau, J. S. (2017). Testing a continuum structure of selfdetermined motivation: A meta-analysis. Psychological Bulletin, 143(12), 1346-1377. https://doi.org/10.1037/bul0000125

Júnior, N., Vissoci, J., Balbim, G., Moreira, C., Pelletier, L., \& Vieira, L. (2014). Crosscultural adaptation and psychometric proprieties analysis of the sport motivation scale - II for the Brazilian context. Revista da Educação Física/UEM, 25(3), 441-458. https://doi.org/10.4025/reveducfis.v25i3.24855

Kline, R. (2016). Methodology in the social sciences. Principles and practice of structural equation modeling (4th ed.). Guilford Press.

Li, C., Kawabata, M., \& Zhang, L. (2018). Validity and reliability of the Sport Motivation Scale-II for Chinese athletes. International Journal of Sport and Exercise Psychology, 16(1), 51-64. https://doi.org/10.1080/1612197X.2016.1153130

Manouchehri, J., Tojari, F., \& Soltanabdl, S. (2015). Validity and Reliability of Measurement Instrument for Sport Motivation Scale in Professional Athletes in Team Sports of Iran. Journal of Psychiatry, 18(2), 1-4. https://doi.org/10.4172/ 2378-5756.1000253

Marsh, H., Morin, A., Parker, P., \& Kaur, G. (2014). Exploratory structural equation modeling: An integration of the best features of exploratory and confirmatory factor analysis. Annual Review of Clinical Psychology, 10(1), 85-110. https://doi.org/10.1146/ annurev-clinpsy-032813-153700

Marsh, H., Hau, K., \& Wen, Z. (2004). In search of golden rules: Comment on hypothesis testing approaches to setting cutoff values for fit indexes and dangers in 
overgeneralizing Hu and Bentler's (1999) findings. Structural Equation Modeling: A Multidisciplinary Journal, 11(3), 320-341. https://doi.org/10.1207.s15328007sem1103_2

Monteiro, D., Cid, L., Teixeira, D., Fonseca, T., Duarte-Mendes, P., Silva, L., \& Rodrigues, F. (2020). Understanding needs satisfaction and frustration in young athletes: Factor structure and invariance analysis. International Journal of Environmental Research and Public Health, 17, 4046. https://doi.org/10.3390/ijerph17114046

Monteiro, D., Teixeira, D., Vitorino, A., Moutão, J., Rodrigues, F., Machado, S., \& Cid, L. (2019). Behavioral Regulation Sport Questionnaire: Gender and sport invariance in Portuguese athletes. Perceptual and Motor Skills, 126(2), 323-341. https://doi.org/10. $1177 / 0031512519825700$

Monteiro, D., Marinho, D., Moutão, J., Couto, N., Antunes, R., \& Cid, L. (2017). Adaptation and validation of the Portuguese version of Basic Psychological Needs Exercise Scale (BPNESp) to the sport domain and invariance across football and swimming. Motricidade, 12(4), 51. https://doi.org/10.6063/motricidade.9372

Muthén, L., \& Muthén, B. (2010). Mplus user's guide. Muthén \& Muthén.

Ntoumanis, N., \& Mallett, C. J. (2014). Motivation in sport: A self-determination theory perspective. In A. G. Papaioannou \& D. Hackfort (Eds.), International perspectives on key issues in sport and exercise psychology. Routledge companion to sport and exercise psychology: Global perspectives and fundamental concepts. (pp. 67-82). Routledge/ Taylor \& Francis Group.

Ntoumanis, N. (2012). A self-determination theory perspective on motivation in sport and physical education: Current trends and possible future research directions. In G. C. Roberts and D. C. Treasure (Eds.), Motivation in sport and exercise. Human Kinetics. (pp. 91-128).

Öcal, K., \& Sakall, D. (2018). Turkish adaptation of the sport motivation scale II (SMSII): Procedures of validity and reliability. International Journal of Sport Exercise and Training Sciences, 4, 39-48. https://doi.org/http://dx.10.18826/useeabd.327789

Pineda-Espejel, H., Alarcón, E., López-Ruiz, Z., Trejo, M., \& Chávez, C. (2016). Propiedades psicométricas de la Escala de Motivación en el Deporte revisada (SMS-II) adaptada al español hablado en méxico. RICYDE. Revista Internacional de Ciencias Del Deporte, 12(44), 107-120. http://dx.doi.org/10.5232/ricyde2016.04402

Pelletier, L., Rocchi, M., Guertin, C., Hébert, C., \& Sarrazin, P. (2019). French adaptation and validation of the Sport Motivation Scale-II (echelle de motivation dans les Sports-II). International Journal of Sport and Exercise Psychology, 17(3), 232-249. https://doi.org/10.1080/1612197X.2017.1339729

Pelletier, L., Rocchi, M., Vallerand, R., Deci, E., \& Ryan, R. (2013). Validation of the revised sport motivation scale (SMS-II). Psychology of Sport and Exercise, 14(3), 329-341. https://doi.org/10.1016/j.psychsport.2012.12.002

Pelletier, L., Fortier, M., Vallerand, R., Tuson, K., Brière, N., \& Blais, M. (1995). Towards a new measure of intrinsic motivation, extrinsic motivation, and amotivation in sports: The Sport Motivation Scale (SMS). Journal of Sport and Exercise Psychology, 17(1), 35-53. https://doi.org/10.1123/jsep.17.1.35

Raykov, T., Gabler, S., \& Dimitrov, D. (2016). Maximal reliability and composite reliability: Examining their difference for multicomponent measuring instruments using latent variable modeling. Structural Equation Modeling: A Multidisciplinary Journal, 23(3), 384-391. https://doi.org/10.1080/10705511.2014.966369 
Rocchi, M., \& Pelletier, L. (2018). How does coaches' reported interpersonal behavior align with athletes' perceptions? Consequences for female athletes' psychological needs in sport. Sport, Exercise, and Performance Psychology, 7(2), 141-154. https:// doi.org/10.1037/spy0000116

Rodrigues, F., Cid, L., Teixeira, D., \& Monteiro, D. (2021). Re-applying the basic psychological needs in exercise scale to exercise groups: Analysis of bifactor models and contextual invariance. Perceptual and Motor Skills, 128(4), 1660-1683. https://doi.org/ $10.1177 / 00315125211016803$

Rodrigues, F., Macedo, R., Cid, L., Teixeira, D., Marinho, D., \& Monteiro, D. (2020). Sex differences in relationships between perceived coach-induced motivational climates, basic psychological needs and behavior regulation among young swimmers. Perceptual and Motor Skills, 127(5), 891-911. https://doi.org/10.1177/0031512520926805

Ryan, R., \& Deci, E. (2017). Self-determination theory: Basic psychological needs in motivation, development, and wellness. Guilford Press.

Ryan, R., \& Connell, J. (1989). Perceived locus of causality and internalization: Examining reasons for acting in two domains. Journal of Personality and Social Psychology, 57(5), 749-761. https://doi.org/10.1037//0022-3514.57.5.749

Sarrazin, P., Vallerand, R., Guillet, E., Pelletier, L., \& Cury, F. (2002). Motivation and dropout in female handballers: A 21-month prospective study. European Journal of Social Psychology, 32(3), 395-418. https://doi.org/10.1002/ejsp.98

Standage, M., \& Ryan, R. (2020). Self-determination theory in sport and exercise. In G. Tanenbaum \& R. C. Eklund (Eds.), Handbook of sport psychology (4th ed.). (pp. 37-56). John Wiley.

Standage, M., Curran, T., \& Rouse, P. (2019). Self-determination-based theories of sport, exercise, and physical activity motivation. In: T. Horn \& A. Smith (Eds.), Advances in sport and exercise psychology. Human Kinetics. https://doi.org/10.1002/ 9781119568124.ch3

Vlachopoulos, S. P., Asci, F. H., Cid, L., Ersoz, G., González-Cutre, D., MorenoMurcia, J. A., \& Moutão, J. (2013). Cross-cultural invariance of the basic psychological needs in exercise scale and need satisfaction latent mean differences among Greek, Spanish, Portuguese and Turkish samples. Psychology of Sport and Exercise, 14(5), 622-631. https://doi.org/10.1016/j.psychsport.2013.03.002

Vlachopoulos, S., \& Michailidou, S. (2006). Development and initial validation of a measure of autonomy, competence, and relatedness in exercise: The basic psychological needs in exercise scale. Measurement in Physical Education and Exercise Science, 10(3), 179-201. https://doi.org/10.1207/s15327841mpee1003_4

\section{Author Biographies}

Filipe Rodrigues, is an adjunct lecturer at Escola Superior de Desporto de Rio Maior (ESDRM -IPSantarém) and researcher at the CIEQV. His research focuses on motivational and cognitive theories to understand health-related behavior change in diverse domains. Other areas of research interest include health psychology, interpersonal behaviors, coaching and neuropsychology.

Luc Pelletier, has been a professor of psychology at the School of Psychology at the University of Ottawa since 1989, where he is full professor since 1997. He completed his doctoral studies in psychology at the University of Quebec in Montreal from 1984 to 1989. His work, guided by the 
Self-Determination Theory, revolves around the intra-personal and interpersonal mechanisms underlying the regulation of motivation, the factors leading to different forms of behavioral motivation as well as the mechanisms underlying change processes. On the applied level, he is interested in motivation for different activities including motivation for health-related behaviors (sports, physical activities, eating) and ecological behaviors.

\section{Rocchi}

Luis Cid, holds a PhD in Sport Sciences (since December 2010), is a professor at the Sport Science School of Rio Maior of the Polytechnic Institute of Santarem (since September 2004) and a senior researcher of the Research Center in Sport, Health and Human Development (since January 2011). Currently, he is the director of the School (since December 2018). Previously he was the subdirector of the school (April 2015-November 2018) and Scientific Coordinator of sports and exercise psychology department (March 2014-April 2018). His academic research field is linked to motivational determinants in sport, physical activity, and healthy lifestyles.

Diogo Teixeira, is a young professor and researcher with a multidisciplinary background and professional practice in several areas of his academic training. He has a degree in Physical Education and Sport; a postgraduation degree in Health, Nutrition, and Exercise; a master's degree in Exercise and Wellness; and a PhD degree in Physical Activity and Health. His research focuses on motivational and emotional determinants and their relationship with the quality of practice in several health and sport contexts.

Diogo Monteiro, holds a PhD in Sports Science and is a professor at the ESECS -Polytechnique of Leiria and is an integrated member of the Research Center in Sport, Health and Human Development. He has a degree in Sport and Exercise Psychology and master's degree in Sport and Exercise Psychology. His academic/research field is linked to motivational determinants in sport and exercise and behavioral change, with a special focus on sedentary behavior, physical activity, healthy lifestyles, well-being, exercise adherence, sport dropout, and persistence. 